\title{
Determination of the first ionization potential of actinium
}

\author{
J. Roßnagel, ${ }^{1, *}$ S. Raeder, ${ }^{1,2}$ A. Hakimi, ${ }^{1}$ R. Ferrer, ${ }^{3}$ N. Trautmann, ${ }^{4}$ and K. Wendt ${ }^{1}$ \\ ${ }^{1}$ Institut für Physik, Universität Mainz, D-55128 Mainz, Germany \\ ${ }^{2}$ TRIUMF, 4004 Wesbrook Mall, Vancouver, BC V6T 2A3, Canada \\ ${ }^{3}$ Instituut voor Kern- en Stralingsfysica, K.U. Leuven, B-3001 Leuven, Belgium \\ ${ }^{4}$ Institut für Kernchemie, Universität Mainz, D-55128 Mainz, Germany
}

(Received 19 December 2011; published 31 January 2012)

\begin{abstract}
Using resonance ionization spectroscopy the first-ionization potential of actinium has been determined by analyzing different Rydberg series in two-color resonant laser excitation. Three individual Rydberg series were investigated, converging toward the ionic ground state and toward the first- and second-excited state of the actinium ion, respectively. A combined analysis of the convergence limits leads to a consistent value for the first-ionization potential of Ac of 43394.45 (19) $\mathrm{cm}^{-1}$, equivalent to $5.380226(24) \mathrm{eV}$.
\end{abstract}

DOI: 10.1103/PhysRevA.85.012525

PACS number(s): 32.10.Hq, 32.80.Ee, 32.80.Rm

\section{INTRODUCTION}

The ionization potential (IP) is a fundamental quantity of each element and defines the element's physical properties and chemical behavior. From an accurate determination of the IP, systematic trends in binding energies for the neighboring elements and valuable information concerning the individual atomic structure can be derived. In heavy multielectron atoms with more than one open valence electron shell, as it is the case for the actinide elements, theoretical calculations on atomiclevel schemes and resulting excitation spectra become more and more difficult and results are often only in poor agreement with experimental data [1-3]. An accurate experimental value of the IP, representing the convergence limit for most of the series of atomic levels for increasing principal quantum number $n$, hence, might serve as a first but stringent test for the suitability of such theoretical estimations. Besides actinium, the IP was precisely determined for nine elements of the actinide series, from thorium up to einsteinium with the exception of protactinium, by using different techniques of laser spectroscopy [4-8]. In general, the most accurate values for the IP were achieved by analyzing convergence limits of Rydberg series [9-11], but a rather high number of atoms was required for the extensive investigations to precisely identify the energetic position of numerous highlying atomic levels. Owing to the high radiation level of the heavier actinide elements, this is not recommendable and experimentally no longer suitable nowadays. In addition, the spectroscopic approach becomes more and more difficult along the sequence of actinide elements, which result from perturbations of the Rydberg series by the increasingly complex structure in heavy multielectron atoms associated to multiple open electron shells. Correspondingly, Köhler et al. [12] and Erdmann et al. [8] analyzed ionization thresholds in external electrical fields applying the saddle-point model by extrapolation of observed field-dependent ionization threshold values to zero field strength. This approach could be carried out experimentally with as few as $10^{12}$ atoms and gave access to the artificially produced actinide elements as rare as einsteinium. The name-giving element of the actinide series, actinium with

*j.rossnagel@uni-mainz.de
$Z=90$, has also been studied with this technique in 2001 by Waldek et al. [13,14], yielding a value of $43398(3) \mathrm{cm}^{-1}$, which has not been confirmed yet. The theoretical expectation value for the IP of Ac scatters over a wide range from 41680 to $45850 \mathrm{~cm}^{-1}$, depending on the extrapolation or calculation methods used [12,15-18].

Actinium is a radioactive element with one long-lived isotope, ${ }^{227} \mathrm{Ac}\left(t_{1 / 2}=28 \mathrm{yr}\right)$, which makes experimental access rather difficult. For the spectroscopic studies presented here just three samples with amounts of only $5 \times 10^{11}$ atoms of ${ }^{227} \mathrm{Ac}$ were available, so that high sensitivity and detection efficiency were prerequisites for the spectroscopic approach. Resonance ionization spectroscopy (RIS) meets these requirements particularly well when applied inside a hot cavity $[11,19,20]$ and when using state-of-the-art pulsed high-power, high-repetition-rate solid-state laser systems [21]. In addition, Ac has a closed $7 s$ shell and only one single electron in the $6 d$ orbital leading to a comparatively simple level scheme with only little perturbations. Thus, even with the described ${ }^{227} \mathrm{Ac}$ samples, extensive investigations on three Rydberg series in the spectrum of Ac I could be performed. The series converges toward the ground state and the first- and second-excited state of the Ac ion, respectively. Apart from further data on these series, a combined analysis using the extended RydbergRitz formalism consistently delivered a precise value for the IP of Ac.

\section{EXPERIMENTAL SETUP}

In RIS experiments the sample atoms are excited stepwise along strong optical transitions by resonant absorption of laser radiation into high-lying atomic levels. From there the atoms are ionized by different processes and the number of ions is detected (e.g., as a function of the frequency of one of the lasers). For resonant excitation of Ac atoms a system of two spectrally tunable pulsed Ti:sapphire lasers was used, which was developed at the university of Mainz [21]. Operated at a repetition rate of $5 \mathrm{kHz}$, each Ti:sapphire laser provided an average output power of up to $4 \mathrm{~W}$, a spectral bandwidth of about $3 \mathrm{GHz}$, and a pulse duration of $40 \mathrm{~ns}$. Using a birefringent filter and an etalon for frequency selection, the laser wavelength can be set in the range from 690 to $960 \mathrm{~nm}$. Alternatively a grating assisted resonator design can 


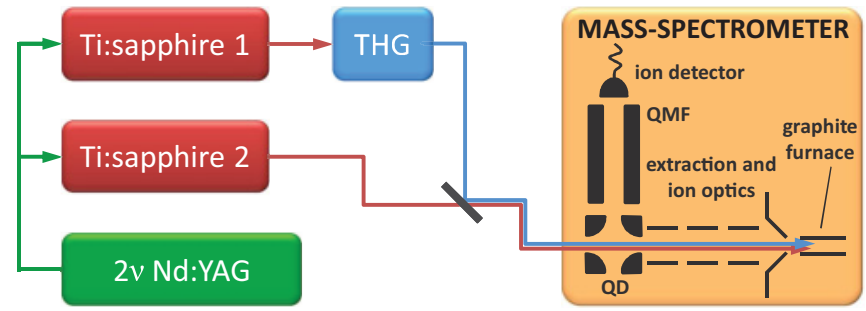

FIG. 1. (Color online) Schematic of experimental setup, including laser system and mass spectrometer. The Ti:sapphire lasers are pumped by a frequency-doubled Nd:YAG laser. A setup for resonant excitation involving frequency-tripled (THG) and fundamental laser radiation is shown. Another setup employing frequency doubling for both Ti:sapphire lasers was also used.

be used, to ensure mode-hop-free laser scans over the whole spectral range with the drawback of slightly lowering the output power. The latter was used to perform extended spectral scans, which were necessary for the identification of Rydberg series. To access the blue and ultraviolet spectral region, second and third harmonics of the laser frequency are attained by single-pass generation through nonlinear optically active crystals (beta barium borate, $\mathrm{BBO}$ ) using variable angle-tuned phase matching. As schematically shown in the experimental setup in Fig. 1, the individual laser beams were overlapped and focused for direct irradiation of the sample atoms within the graphite furnace of the mass spectrometer. Therein an efficient atomization of actinium was achieved by resistively heating the furnace to temperatures of about $1600{ }^{\circ} \mathrm{C}$. Since every atom is exposed to many successive laser pulses before leaving the cavity, RIS within the hot furnace ensures high excitation and ionization efficiencies. By properly tuning the lasers, the Ac atoms were excited stepwise into even-parity Rydberg states lying below as well as above the first-ionization potential. Highly excited atoms in Rydberg states below the first-ionization potential were ionized by blackbody radiation, additional laser photons or collisions inside the hot cavity, while atoms in Rydberg states above the first-ionization potential basically undergo autoionization via the coupling to the underlying continuum channel. The generated ions were guided toward the exit hole of the cavity by the heating potential and extracted by moderate electrostatic fields of below $100 \mathrm{~V} / \mathrm{cm}$. After collimation and beam shaping by ion optics, an electrostatic quadrupole deflector (QD) separated the ion beam from all kinds of neutral particles emitted from the hot cavity which otherwise would cause a background signal. Subsequent mass separation in a radiofrequency quadrupole mass filter (QMF) operating at $1.2 \mathrm{MHz}$ [22] provided an isotopic abundance sensitivity exceeding $10^{-5}$ in the mass range of the actinides and secured the suppression of thermal surface ions of other elements emitted from the furnace. Finally, the mass-selected ions were detected in a channel electron multiplier operating in single-ion counting mode.

\section{RESULTS AND DISCUSSION}

To observe and identify suitable Rydberg series altogether twelve atomic levels with odd parity in the energy range from $21195 \mathrm{~cm}^{-1}$ to $33674 \mathrm{~cm}^{-1}$, taken from the literature $[23,24]$, were tested as first-excited states (FES) and led to (a)

(b)

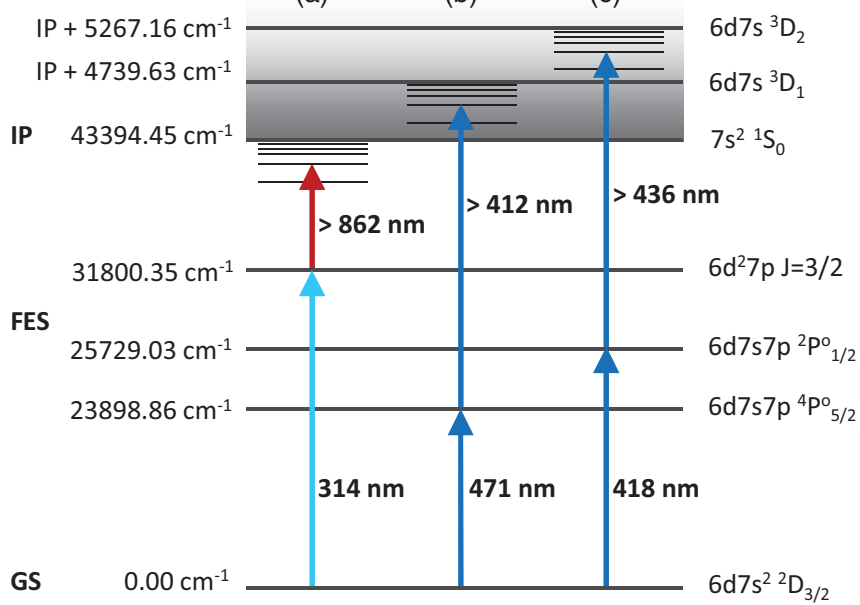

FIG. 2. (Color online) Excitation schemes used for the resonant ionization of Ac via Rydberg states; for precise wavelengths, see text. The energetic positions of the atomic levels as well as the assignment of their configurations are taken from the literature $[23,24]$ and are given with an error of typically less than $0.01 \AA$.

the identification of three strong and thus useful transitions for resonant ionization. In all cases saturation powers of below $20 \mathrm{~mW}$ were measured in the experimental geometry with the laser parameters discussed above and were easily reached with the available laser power. From each of these three FESs, Rydberg series in Ac could be measured for the first time by applying long-range scans of the frequency by the grating-assisted Ti:sapphire laser. Figure 2 shows the corresponding two-step excitation schemes together with the convergence limits of the individual Rydberg series.

For observation of the first Rydberg series converging to the ground state of the Ac ion, the atoms were transferred from the atomic ground state with the configuration $6 d 7 s^{2}{ }^{2} D_{3 / 2}$ to a $6 d^{2} 7 p J=3 / 2$ (odd-parity) state at $31800.35 \mathrm{~cm}^{-1}$ by resonant third-harmonic laser radiation of $314.462 \mathrm{~nm}$. From this state about 40 members of the bound Rydberg series were excited in the range of 858 to $889 \mathrm{~nm}$ by fundamental radiation of the grating-assisted Ti:sapphire laser. Figure 3(a) shows the measured ion signal of this scan as a function of the total excitation energy. Altogether 37 Rydberg resonances were identified and indicated by their most probable principal quantum number $n$. As no conclusive assignment of the series can be derived, these values of $n$ were determined by minimizing the quantum defect. A possible variation of adding an integer will neither affect the fitting process nor the result of the IP being the convergence limit of the series. In the range of resonances with low principal quantum numbers $n=20$ to $n=30$, a significant splitting of peaks into individual resonances is observed. This corresponds to a splitting into different electron-core configurations because there are no observable interferences between these resonances. The collected data, however, are not sufficient for any conclusive analysis.

The Rydberg series to the first-excited state of the ion at $4739.63 \mathrm{~cm}^{-1}$ above the IP having a $6 d 7 s^{3} D_{1}$ configuration was excited from the $6 d 7 s 7 p^{4} P_{5 / 2}^{o}$ state at $23898.86 \mathrm{~cm}^{-1}$ by tuning the frequency-doubled laser radiation between 

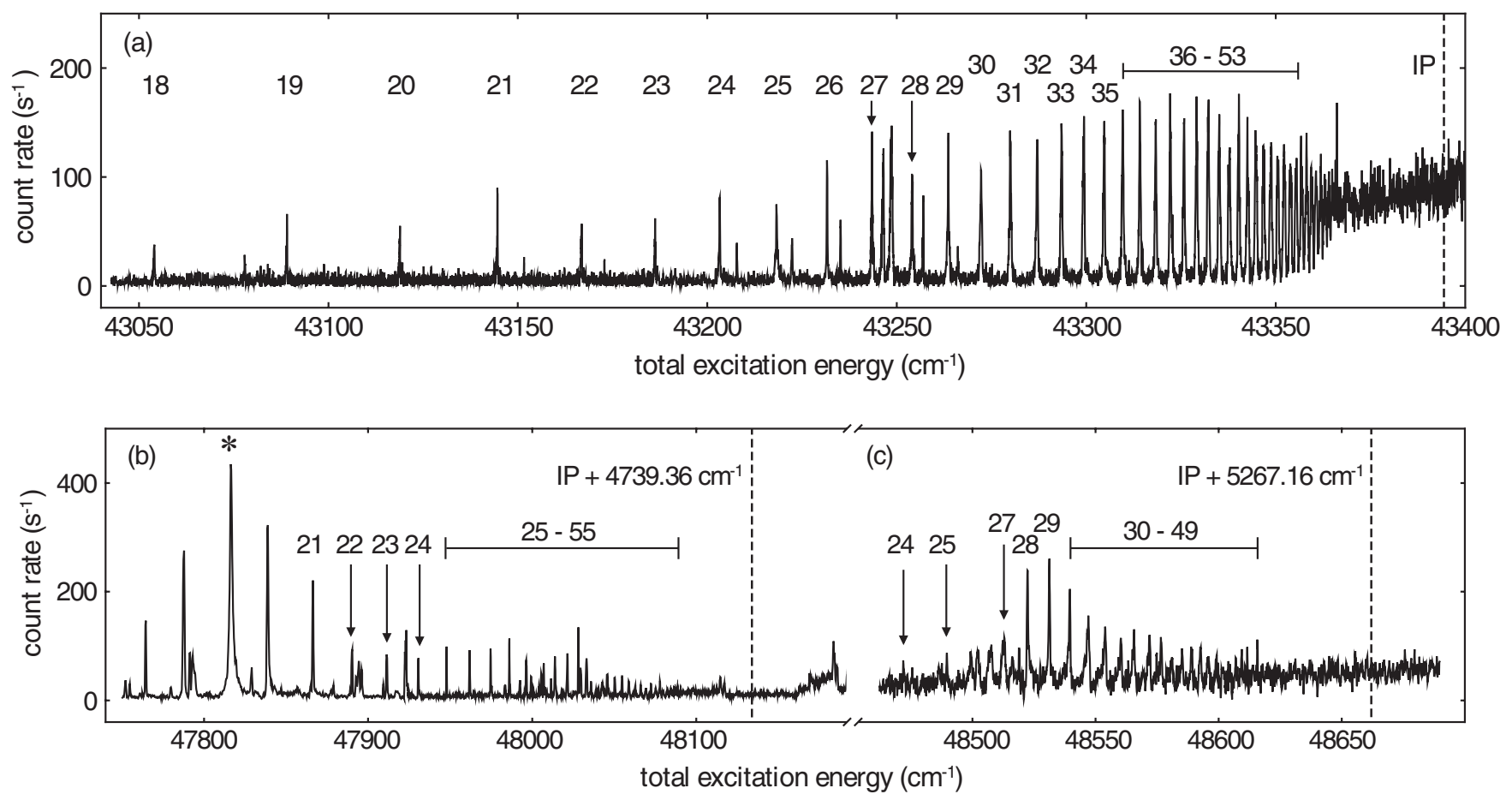

FIG. 3. Top: observed Rydberg series to ionic ground state with given principal quantum numbers (a); see text for assignment. The Rydberg states shown were excited from the intermediate level at $31800.35 \mathrm{~cm}^{-1}$. Bottom: the two Rydberg series to the (b) first- and (c) second-excited states of the ion, excited from the levels at $23898.86 \mathrm{~cm}^{-1}$ and $25729.03 \mathrm{~cm}^{-1}$, respectively (cf. Fig. 2).

412 and $418 \mathrm{~nm}$. The corresponding intermediate level was populated using frequency-doubled laser radiation at a wavelength of $471.790 \mathrm{~nm}$. In the resulting spectrum in Fig. 3(b), in addition to the 39 members of the Rydberg series, strong interference patterns can be observed in the form of a multitude of interloping resonances and broadened and asymmetric peaks, which are due to the competing ionization channels from the autoionizing Rydberg series and the underlying ionization continuum. These are typical phenomena for Rydberg series lying above the first-ionization potential $[25,26]$ and can be modeled in the framework of multichannel quantum defect theory (MQDT) [27,28]. In this Rydberg series a severe disturbance at the lower end of the series is obvious. A strong interloper is overlaying the resonance corresponding to $n=19$, which is marked by an asterisk. The neighboring resonances show typical asymmetric peak profiles and are shifted up to $6 \mathrm{~cm}^{-1}$ from their predicted positions. For this reason these resonances were not taken into account for the subsequent evaluation.

The Rydberg series to the second state of the ion at $5267.16 \mathrm{~cm}^{-1}$ above the IP, shown in Fig. 3(c), reveals even stronger interferences causing severe asymmetries of the peak shapes and large deviations of the resonances from their expected positions, caused by the interaction of two underlying ionization continua and interloping resonances. For the spectroscopy of this series an excited atomic level at $25729.03 \mathrm{~cm}^{-1}$ with configuration $6 d 7 s 7 p^{2} P_{1 / 2}^{o}$ was populated from the ground state by laser radiation of $418.430 \mathrm{~nm}$ from which the Rydberg states could be excited by frequency-doubled laser light in the range of 436 to $441 \mathrm{~nm}$. In this disturbed series only 26 Rydberg states could be assigned.
Each of the Rydberg resonances was fit individually to obtain its precise energy position. Gaussian line shapes reproduced the resonances of the first series very well, as no asymmetries were observed and the laser linewidth combined with the Doppler broadening was much larger than the natural linewidth of typical Rydberg resonances. Autoionizing resonances above the first-ionization potential typically showed asymmetric line shapes [25,26], so that a Gaussian approximation was not sufficient. For these resonances Fano profiles [29] of the form

$$
I\left(q, \gamma, E_{0}\right)=\frac{\left[q \frac{\gamma}{2}+\left(E-E_{0}\right)\right]^{2}}{\left(\frac{\gamma}{2}\right)^{2}+\left(E-E_{0}\right)^{2}}
$$

were used, as claimed by the MQDT, in good agreement with the experimental data. Here the shape parameter $q$ represents the asymmetry of a resonance with a spectral linewidth $\gamma$. For the total energetic position of the individual resonances, the excitation energy is summed up with the observed energy of the first-excited states, which was found to be in all three cases in good agreement with the corresponding literature value.

The value for the IP can be extracted from the individual positions $E_{n}$ of the resonances of one series with high precision using the Rydberg-Ritz formula

$$
E_{n}=E_{\mathrm{IP}}-\frac{R_{\mu}}{[n-\delta(n)]^{2}} .
$$

This formula describes the energy positions of the resonances with principal quantum numbers $n$, converging to the limit of the series $E_{\mathrm{IP}}$ for $n \rightarrow \infty$. $R_{\mu}$ describes the mass-reduced 
Rydberg constant while $\delta(n)$ is the $n$-dependent quantum defect, which can be described by the Ritz expansion [30,31]

$$
\delta(n)=A+\frac{B}{(n-A)^{2}}
$$

with sufficient precision. In this expansion $A$ is an $n$ independent constant part of the quantum defect and $B$ is the slope of the form factor which depends on the configuration of the series. Further expansions and possible stark shifts can be neglected owing to the large spectral linewidth of the lasers of $3 \mathrm{GHz}$ and only small electric fields of less than $1 \mathrm{~V} / \mathrm{cm}$ within the graphite furnace.

In Fig. 4 the convergence limit of the excitation energies of the observed resonances for the three Rydberg series are shown with fits from the Rydberg-Ritz formula (2) and the corresponding residuals. Huge differences in the absolute values of the residuals between the series converging to the ground state and those to the excited states are obvious and reflect the degree of perturbation of the individual series. As already mentioned, such perturbations in Rydberg series cause shifts in

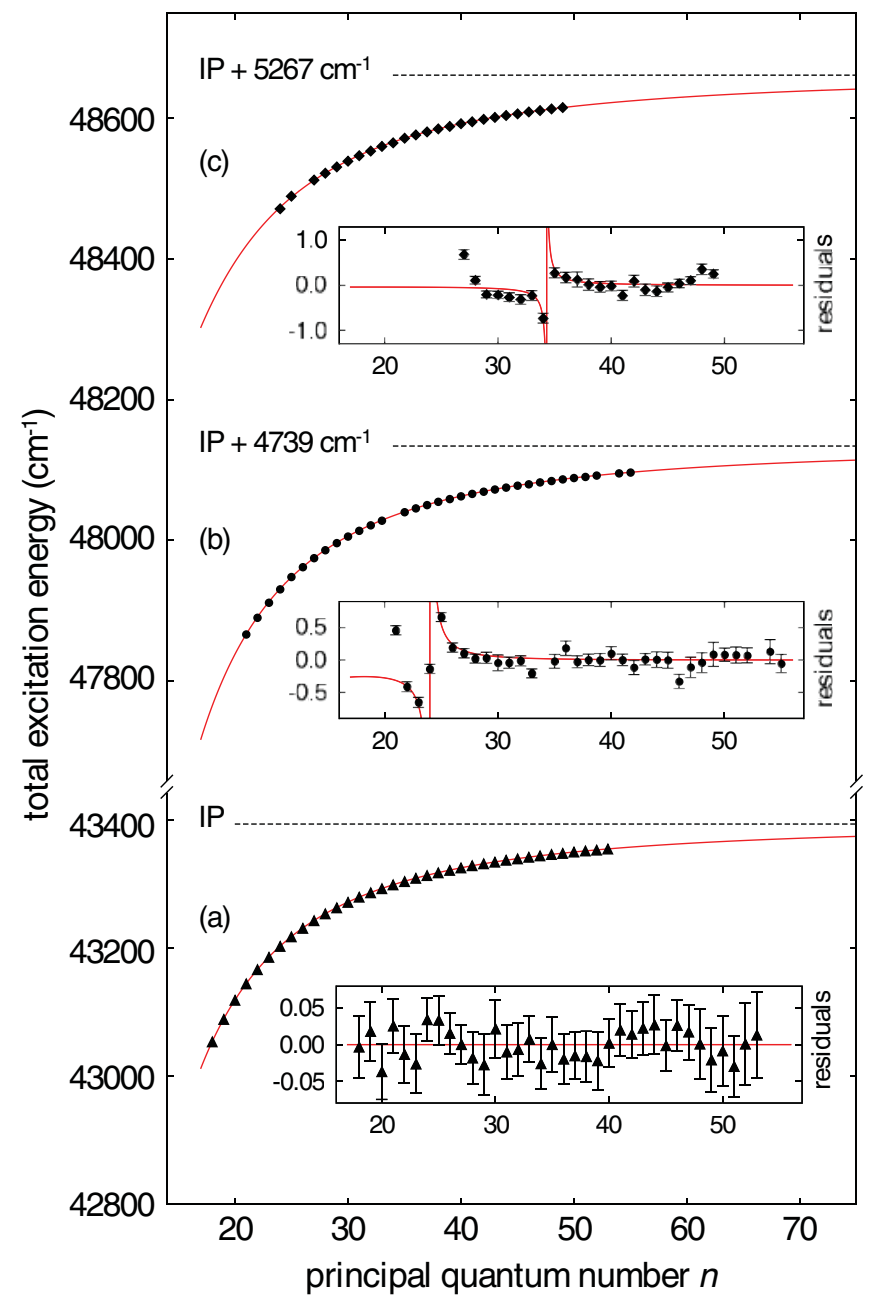

FIG. 4. (Color online) Convergences with Rydberg-Ritz fits of individual Rydberg series (a) to the ground state of the ion, (b) to the first-excited state at IP $+4739.63 \mathrm{~cm}^{-1}$, and (c) to the second-excited state at IP $+5267.16 \mathrm{~cm}^{-1}$. The insets show the residuals of the corresponding series including the MQDT corrections. the positions of the peaks of the observed Rydberg resonances due to interference effects that have to be considered for the calculation of the IP. In the framework of the MQDT, these effects can be taken into account by an expansion of the undisturbed quantum defect $\delta_{0}(n)$ from Eq. (3) by

$$
\delta(n)=\delta_{0}(n)-\frac{1}{\pi} \arctan \left(\frac{\Gamma_{j} / 2}{E-E_{j}}\right),
$$

where $E_{j}$ is the position of an interloper with a spectral width $\Gamma_{j}$ [28]. These corrections are shown in the residuals in Fig. 4 and are taken into account for the calculation of the IP. The error bars of the residuals include statistical errors from the fitting routine as well as systematic uncertainties from the wavelength determination. Likewise, an uncertainty for the wavelength of the first-excited state was taken into account.

For Rydberg series lying below the first ionization limit the oscillator strength for photoexcitation decreases rapidly with increasing $n$ as $n^{-3}$ [32]. This trend can be observed in the intensity distribution of the Rydberg states with high $n$ in the series converging to the ground state of the Ac ion shown in Fig. 3(a). For lower $n$, a smaller intensity of the ion signal is obvious because of the reduced ionization probabilities of these states due to collisions and blackbody radiation. This series has been measured two times and has shown the same characteristics.

In the second series toward the first-excited state of the Ac ion, an analysis of the intensities of the Rydberg states is difficult because of the strong interferences by the interloping resonances. The interaction of these resonances with neighboring Rydberg states [exemplary for the interloper marked with an asterisk in Fig. 3(b)] causes strong deviations in the intensity distribution.

For two interfering ionization continua, as is the case for the Rydberg series converging to the second ionic state in Fig. 3(c), not only are the peak shapes of the Rydberg resonances asymmetric, but the envelope for the ionization probability also follows a Fano profile (1) $[33,34]$, which is shown in Fig. 5. In the corresponding plot of the residuals in Fig. 4, an additional interloping resonance can be inferred from the jump in the residuals at an excitation energy of $48569 \mathrm{~cm}^{-1}$.

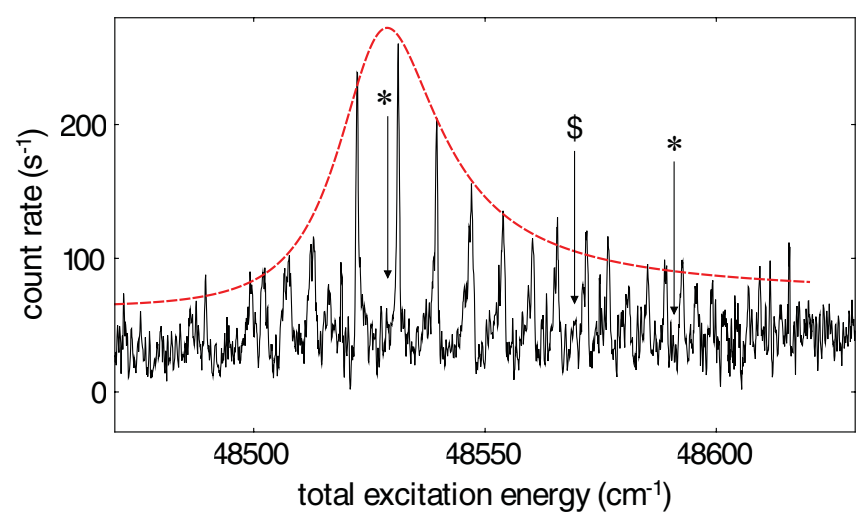

FIG. 5. (Color online) Enlargement of the Rydberg series converging to the ionic level at IP $+5267.16 \mathrm{~cm}^{-1}$ from Fig. 3(c). The dashed line represents the fit envelope function of the ionization probability. The position of the interloper is marked by a dollar sign and the two points of $q$ reversal are indicated by an asterisk. 
TABLE I. Convergence limits for the individual Rydberg series and resulting values for ionization potential $E_{\mathrm{IP}}$ from known excitation energies $E_{\text {exc }}$ for the ionic states. For the convergence limits, purely statistical errors are given. The errors for resulting IP values include systematic and statistical uncertainties; the two results for the first series were merged to a weighted average value. The final number is the weighted averege of the individual results.

\begin{tabular}{lcc}
\hline \hline Limit $\left[\mathrm{cm}^{-1}\right]$ & Ac II $E_{\mathrm{exc}}\left[\mathrm{cm}^{-1}\right]^{1}$ & $E_{\mathrm{IP}}\left[\mathrm{cm}^{-1}\right]$ \\
\hline $43394.530(3)$ & 0 & \\
$43394.65(10)$ & 0 & $43394.54(28)$ \\
$48133.88(27)$ & $4739.63(11)$ & $43394.25(34)$ \\
$48661.75(43)$ & $5267.16(11)$ & $43394.59(45)$ \\
& & $43394.45(19)$ \\
\hline
\end{tabular}

This interloper is barely observable; therefore, in Fig. 5 it is marked by a dollar sign. Beside the Fano-like peak shapes, the interference of the Rydberg series with this interloper and two ionization channels causes two points of $q$ reversal, where the shape parameter $q$ changes its sign [35-37]. Consequently, the direction of asymmetry of the peak shapes changes its direction, as shown in Fig. 5. Hence, the behavior of this Rydberg series follows stringently the MQDT predictions, but nevertheless these disturbances reduce the total accuracy for the extraction of the IP value.

The results for the convergence limits of the observed Rydberg series are summarized in Table I. The IP values can be extracted from the individual limits via the known excitation energies of the ionic levels from arc spectra analysis [23,24], which are independent of the particular value of the IP. The error of these energetic positions can be derived from the cited literature to be $0.11 \mathrm{~cm}^{-1}$ for these transitions and is taken into account for the determination of the IP. The resulting values for the IP from the discussed series are consistent within their uncertainty range. Nevertheless, it is obvious that, as discussed above, the statistical uncertainty of the unperturbed series converging to the ground state of the Ac ion is about one order of magnitude smaller than those of the higher-lying series. Because of the unknown configurations of the discussed Rydberg series, $\delta(n)$ is set to be the minimal positive value, which gives rise to the principal quantum numbers $n$ shown in Fig. 3. As mentioned before, the ambiguous value of $n$ neither
TABLE II. Comparison between our result for the IP of Ac and literature values obtained by extrapolation, $a b$ initio calculations, or by experiment, given in chronological order. Errors are given when available. The difference between these values and ours is given, as well as the method used and the corresponding references.

\begin{tabular}{lcccc}
\hline \hline$E_{\mathrm{IP}}\left[\mathrm{cm}^{-1}\right]$ & Diff. $\left[\mathrm{cm}^{-1}\right]$ & Method used & Year & Ref. \\
\hline $41700(1000)$ & -1700 & Extrapol. & 1973 & {$[16]$} \\
45730 & 2336 & Extrapol. & 1978 & {$[17]$} \\
45849 & 2455 & Extrapol. & 1997 & {$[12]$} \\
42900 & -450 & Calc. & 1998 & {$[18]$} \\
$43398(3)$ & 3.5 & Expt. & 2001 & {$[13]$} \\
42699 & -695 & Calc. & 2010 & {$[15]$} \\
$43394.45(19)$ & 0 & Expt. & 2011 & (this work) \\
\hline \hline
\end{tabular}

affects the precision nor the value of the convergence limit of the series.

A weighted average of the individual measurements gives a resulting value for the IP of Ac of 43394.45 (19) $\mathrm{cm}^{-1}$, which corresponds to $5.380226(24) \mathrm{eV}$, where the statistical and the systematic errors are considered to be uncorrelated. Compared to the experimental result from Waldek et al., this value is $-3.5 \mathrm{~cm}^{-1}$ lower, slightly exceeding the error bar given therein. As the error of our result is more than a factor of 10 smaller than the one given by Waldek et al., our measurement can be regarded as confirmation and significant refinement of the earlier result. In Table II, a comparison of the different theoretical predictions for the IP value with the experimental results is shown. The huge discrepancies between these values and the wide scattering of the theoretical values reveal the difficulties of these approximation methods in particular heavy multielectron atoms like actinium. As continuation of this work the investigation of other missing IP values is in preparation.

\section{ACKNOWLEDGMENTS}

This research has been financially supported by the German Bundesministerium für Bildung und Forschung (06 MZ 228), by FWO-Vlaanderen (Belgium), GOA/2004/03 (BOF K. U. Leuven), and the IUAP-Belgian State Science Policy (BriX network P6/23). We also thank P. Thörle-Pospiech for the preparation of the Ac samples.
[1] M. Dolg, Encycl. Comput. Chem. 2, 1478 (1998).

[2] W. Liu, W. Küchle, and M. Dolg, Phys. Rev. A 58, 1103 (1998).

[3] B. Judd, Phys. Rev. 125, 613 (1962).

[4] S. Johnson, B. Fearey, C. Miller, and N. Nogar, Spectrochim. Acta, Part B 47, 633 (1992).

[5] E. Worden and J. Conway, J. Opt. Soc. Am. 69, 733 (1979).

[6] E. Worden, L. Carlson, S. Johnson, J. Paisner, and R. Solarz, J. Opt. Soc. Am. B 10, 1998 (1993).

[7] A. Coste, R. Avril, P. Blancard, J. Chatelet, D. Lambert, J. Legre, S. Liberman, and J. Pinard, J. Opt. Soc. Am. 72, 103 (1982).
[8] N. Erdmann, M. Nunnemann, K. Eberhardt, G. Herrmann, G. Huber, S. Köhler, J. Kratz, G. Passler, J. Peterson, N. Trautmann, and A. Waldek, J. Alloys Compd. 271, 837 (1998).

[9] B. Bushaw, K. Blaum, and W. Nörtershäuser, Phys. Rev. A 67, 022508 (2003)

[10] M. Miyabe, C. Geppert, M. Kato, M. Oba, I. Wakaida, K. Watanabe, and K. Wendt, J. Phys. Soc. Jpn. 75 (2006).

[11] C. Mattolat, T. Gottwald, S. Raeder, S. Rothe, F. Schwellnus, K. Wendt, P. Thörle-Pospiech, and N. Trautmann, Phys. Rev. A 81, 052513 (2010). 
[12] S. Köhler, K. Eberhardt, N. Erdmann, G. Herrmann, G. Huber, J. Kratz, M. Nunnemann, G. Passler, P. Rao, J. Riegel, N. Trautmann, and K. Wendt, Spectrochim. Acta, Part B 52, 717 (1997).

[13] A. Waldek, N. Erdmann, C. Gruening, G. Huber, P. Kunz, J. Kratz, J. Lassen, G. Passler, and N. Trautmann, in AIP Conf. Proc., Vol. 584 (2001), p. 219.

[14] E. Worden, J. Blaise, M. Fred, N. Trautmann, J. Wyart, L. Morss, N. Edelstein, and J. Fuger, The Chemistry of the Actinide and Transactinide Elements (Springer, Dordrecht, Netherlands, 2006), p. 1836.

[15] L. Özdemir and G. Uerer, Acta Phys. Pol. A 118, 563 (2010).

[16] J. Sugar, J. Chem. Phys. 60, 4103 (1974).

[17] K. Rajnak and B. Shore, J. Opt. Soc. Am. 68, 360 (1978).

[18] E. Eliav, S. Shmulyian, U. Kaldor, and Y. Ishikawa, J. Chem. Phys. 109, 3954 (1998).

[19] T. Kessler, K. Brueck, C. Baktash, J. Beene, C. Geppert, C. Havener, H. Krause, Y. Liu, D. Schultz, D. Stracener, C. Vane, and K. Wendt, J. Phys. B 40, 4413 (2007).

[20] T. Gottwald, C. Geppert, F. Schwellnus, K. Wies, K. Wendt, Y. Liu, C. Baktash, J. Beene, C. Havener, H. Krause, D. Schulz, D. Stracener, C. Vane, T. Kessler, and B. Tordoff, Nucl. Instrum. Methods Phys. Res., Sect. B 266, 4398 (2008).

[21] C. Mattolat, S. Rothe, F. Schwellnus, T. Gottwald, S. Raeder, and K. Wendt, in AIP Conf. Proc. (2009), Vol. 1104, p. 114.
[22] K. Blaum, C. Geppert, P. Müller, W. Nörtershäuser, E. Otten, A. Schmitt, N. Trautmann, K. Wendt, and B. Bushaw, Int. J. Mass Spectrom. 181, 67 (1998).

[23] W. Meggers, M. Fred, and F. Tompkins, J. Res. Natl. Bur. Stand. 58 (1957).

[24] W. Meggers, Spectrochim. Acta 10, 195 (1957).

[25] W. Cooke and C. Cromer, Phys. Rev. A 32, 2725 (1985).

[26] F. Mies, Phys. Rev. 175, 164 (1968).

[27] U. Fano, J. Opt. Soc. Am. 65, 979 (1975).

[28] M. Seaton, Rep. Prog. Phys. 46, 167 (1983).

[29] U. Fano, Phys. Rev. 124, 1866 (1961).

[30] W. Ritz, Ann. Phys. 317, 264 (1903).

[31] D. Hartree, in Mathematical Proceedings of the Cambridge Philosophical Society (Cambridge University Press, Camebridge, UK, 1928), Vol. 24, p. 426.

[32] J. Connerade, Highly Excited Atoms (Cambridge University Press, Cambridge, UK, 1998).

[33] A. Giusti-Suzor and H. Lefebvre-Brion, Phys. Rev. A 30, 3057 (1984).

[34] S. Cohen, Eur. Phys. J. D 4, 31 (1998).

[35] H. Friedrich, Theoretical Atomic Physics (Springer, Berlin Heidelberg, Germany, 2006).

[36] F. Gounand, T. Gallagher, W. Sandner, K. Safinya, and R. Kachru, Phys. Rev. A 27, 1925 (1983).

[37] J. Connerade, Proc. R. Soc. A 362, 361 (1978). 